EX/S3-2

\title{
MHD Instabilities and Their Effects on Plasma Confinement in the Large Helical Device Plasmas
}

K. Toi, S. Ohdachi, S. Yamamoto ${ }^{\text {, }}$ N. Nakajima, S. Sakakibara, K.Y. Watanabe, S. Inagaki, Y. Nagayama, Y. Narushima, H. Yamada, K. Narihara, S. Morita, T. Akiyama ${ }^{\text {b }}$ N. Ashikawa, X. Ding ${ }^{\text {c }}$, M. Emoto, H. Funaba, M. Goto, K. Ida, H. Idei, T. Ido, K. Ikeda, S. Imagawa, M. Isobe, K. Itoh, O. Kaneko, K. Kawahata, T. Kobuchi, A. Komori, S. Kubo, R. Kumazawa, J. Lid, Y. Liang, S. Masuzaki, T. Mito, J. Miyazawa, T. Morisaki, S. Murakami, S. Muto, T. Mutoh, K. Nagaoka, Y. Nakamura, H. Nakanishi, K. Nishimura, A. Nishizawa, N. Noda, T. Notake ${ }^{\text {a }, ~ K . ~ O h k u b o, ~ I . ~ O h t a k e, ~ N . ~ O h y a b u, ~ Y . ~ O k a, ~ S . ~ O k a m u r a, ~}$ T. Ozaki, B.J. Peterson, A. Sagara, T. Saida ${ }^{\mathrm{e}}$, K. Saito, R. Sakamoto, M. Sasao, K. Sato, M. Sato, T. Satow, T. Seki, T. Shimozuma, M. Shoji, S. Sudo, M.Y. Tanaka, N. Tamura ${ }^{\mathrm{e}}$, K. Tanaka, K. Tsumori, T. Uda, T. Watari, A. Weller ${ }^{\text {f, }, ~ Y . ~ X u, ~ I . ~ Y a m a d a, ~ M . ~ Y o k o y a m a, ~ S . ~ Y o s h i m u r a, ~ Y . ~ Y o s h i m u r a, ~ K . ~ Y a m a z a k i, ~}$ K. Matsuoka, O. Motojima, Y. Hamada, M. Fujiwara

National Institute for Fusion Science, Oroshi-cho 322-6, Toki, 509-5292,Japan

a Department of Energy Engineering and Science, Nagoya University, Nagoya, Japan

${ }^{\mathrm{b}}$ Research Lab. for Nucl. Reactors, Tokyo Inst. Tech., Tokyo 152-8550, Japan

${ }^{c}$ Southwestern Institute of Physics, Chengdu, China

${ }^{\mathrm{d}}$ Institute of Plasma Physics, Akademia Sinica, Hefei, China

${ }^{\mathrm{e}}$ Department of Fusion Science, Graduate University for Advanced Studies, Hayama, Japan

${ }_{\mathrm{f}}^{\mathrm{f}}$ Max-Planck-Institut für Plasmaphysik, Garching, Germany

E-mail: toi@nifs.ac.jp

\begin{abstract}
Characteristics of MHD instabilities and their impacts on plasma confinement are studied in current free plasmas of the Large Helical Device(LHD). Spontaneous L-H transition is often observed in high beta plasmas in the range of $2 \%$ averaged beta at low toroidal field $\left(\mathrm{B}_{\mathrm{t}} \leq 0.6 \mathrm{~T}\right)$. The stored energy rapidly rises by the transition, but quickly saturates due to the growth of $m=2 / n=3$ and $m=2 / n=2$ modes ( $m$ and $n$ : poloidal and toroidal mode numbers) excited in the plasma edge region. Even in low beta plasmas, ELM like activities are sometimes induced in high performance plasmas with steep edge pressure gradient, and transiently reduce the stored energy by about 10\%. Energetic ion driven MHD modes such as Alfven eigenmodes are studied in the very wide range of characteristic parameters: the averaged beta of energetic ions $\left\langle\beta_{\mathrm{b} / /}\right\rangle$ up to $5 \%$ and the ratio of energetic ion velocity to the Alfven velocity $\mathrm{V}_{\mathrm{b} / /} / \mathrm{V}_{\mathrm{A}}$ up to 2.5. In addition to the observation of toroidicity induced Alfven eigenmodes (TAEs), coherent magnetic fluctuations of helicity induced Alfven eigenmodes (HAEs) have been observed for the first time in NBI heated plasmas. Transition of TAE to global Alfven eigenmode(GAE) is also observed in a discharge with temporal evolution of the rotational transform profile, having a similarity to the phenomenon in a reversed shear tokamak. At the low magnetic field, bursting TAEs transiently induce a significant loss of energetic ions, but lead to the transient improvement of bulk plasma confinement in the plasma central region.
\end{abstract}


EX/S3-2

\section{Introduction}

In the Large Helical Device (LHD), high performance plasmas were obtained in so-called "inward-shifted configuration" (the magnetic axis position of the vacuum field: $\mathrm{R}_{\mathrm{ax}} \leq 3.6 \mathrm{~m}$ ) where energetic particles are expected to be well confined[1]. However, the inward-shifted configuration is unfavorable for MHD stability against pressure driven modes because of the presence of magnetic hill region. The unfavorable behaviors can be mitigated by the Shafranov shift, which tends to generate magnetic well in the plasma core region. The edge region, however, is always the magnetic hill. The rotational transform of the configuration at the vacuum field is in the range of $1(0) \sim 0.4$ at the center and t(1) 1.3 at the last closed flux surface(LCFS), respectively. When the averaged toroidal beta $\left\langle\beta_{\mathrm{t}}\right\rangle$ increases, $\mathfrak{l}(0)$ increases and $\mathfrak{l}(1)$ decreases appreciably due to large Shafranov shift. It is crucial for an LHD plasma to clarify impacts of MHD instabilities on confinement of bulk plasma and energetic ions, although serious deterioration of the confinement by MHD modes was not recognized in the past experimental campaigns with up to absorbed NBI power $\left(\mathrm{P}_{\mathrm{abs}} \leq 4 \mathrm{MW}\right)$ and toroidal beta $\left(\left\langle\beta_{\mathrm{t}}\right\rangle \leq 2 \%\right)$ [2]. After that, experiments to investigate achievable $\left\langle\beta_{\mathrm{t}}\right\rangle$ were attempted with up to $6 \mathrm{MW}$ absorbed power. MHD stability of the inward-shifted plasmas was intensively studied in the wide parameter range. Main MHD instabilities observed in LHD are as follows: (1) low n MHD modes destabilized by the pressure gradient in the plasma edge as well as the core region, and (2) energetic ion driven MHD modes such as Alfven eigenmodes (AEs) . In this paper, we discuss characteristics of these MHD modes and their impacts on plasma confinement.

\section{Pressure driven modes and their effects on plasma confinement}

Most dangerous MHD modes in the plasma core region of LHD are low n pressure driven modes such as $m=2 / n=1$ mode(m:poloidal mode number, $n$ : toroidal mode number). When the plasma beta is increased in the inward-shifted configuration $\left(\mathrm{R}_{\mathrm{ax}} \leq 3.6 \mathrm{~m}\right)$, the rotational transform near the center tends to increase and approaches to 0.5 . Then, $m=2 / n=1$ mode becomes more unstable and affects the bulk plasma confinement [2]. A typical example that the averaged toroidal beta $\left\langle\beta_{\mathrm{t}}\right\rangle$ obtained with diamagnetic measurement saturates due to excitation of $m=2 / n=1$ mode is shown in Fig.1, where NBI absorbed power is relatively low $(\sim 3 \mathrm{MW})$. In this shot, the plasma beta is increased rapidly by ice pellet injection and $m=2 / n=1$ is suddenly destabilized when $\left\langle\beta_{\mathrm{t}}\right\rangle$ exceeds about $2.2 \%$. The $m=2 / n=2$ MHD mode is simultaneously destabilized in the plasma edge, together with $m=2 / n=1$ mode. If the rational surface of $\imath=0.5$ is removed by the effects of raising the plasma beta and small amount of net plasma current flowing in the co-direction, $m=2 / n=1$ mode is suppressed and the bulk confinement recovers [2]. When the pressure gradient in the core region is transiently increased by ice

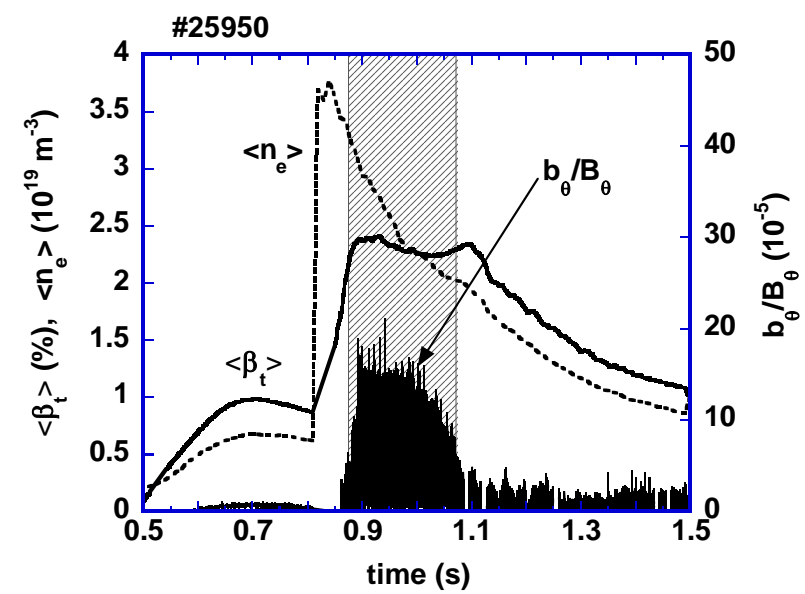

Fig.1 Time evolution of a high beta plasma

affected by $m=2 / n=1$ MHD mode $\left(b_{\theta}\right)$, where $B_{t}=0.6 T$ and $R_{a x}=3.6 m$. An ice pellet is injected at $t=0.85 \mathrm{~s}$. $B_{\theta}$ stands for the averaged poloidal field at the magnetic probe position. 
EX/S3-2

pellet injection, sawtooth oscillations or internal collapses are sometimes induced, accompanying $\mathrm{m}=2 / \mathrm{n}=1$ pressure driven modes even in a fairly low beta plasma $(\sim 1 \%)[3] . \quad$ In the previous experimental campaigns, the pressure profile becomes broad with the increase in the averaged plasma beta [4]. This may be favorable for increasing the beta value if the pressure profile has several local flat zones around the low order rational surfaces [5]. The broadening of the pressure profile in high beta plasmas will be due to anomalous transport and broader heating deposition rather than MHD stability, because no large amplitude MHD modes are observed in the plasma core region. It is crucial whether the edge stability is ensured in a plasma with steep pressure gradient near the edge. The broadening of the pressure profile tends to steepen the edge pressure gradient with the increase in the heating power. When higher NBI power is applied to a high density plasma, the plasma beta rapidly passes through $\sim 2 \%$, and the $m=2 / n=1$ mode will not be excited. However, MHD modes such as $\mathrm{m}=2 / \mathrm{n}=2$ mode related to $\mathrm{l}=1$ rational surface just inside LCFS would be destabilized. In high beta plasmas with more than $\sim 2 \%$ at fairly low toroidal field $\mathrm{Bt}=0.5-0.6 \mathrm{~T}, \mathrm{~L}-\mathrm{H}$ transition is often induced. So far, this transition only takes place in hydrogen plasmas in the configuration of $\mathrm{R}_{\mathrm{ax}}=3.6 \mathrm{~m}$ for more than $\mathrm{P}_{\mathrm{abs}} \sim 3 \mathrm{MW}$. The $\mathrm{L}-\mathrm{H}$ transition provides an excellent opportunity to test the edge stability of LHD plasmas. A typical high beta discharge with L-H transition is shown in Fig.2 (a), where $\mathrm{P}_{\text {abs }}$ $\sim 6 \mathrm{MW}$ at $\mathrm{B}_{\mathrm{t}}=0.5 \mathrm{~T}$. The $\mathrm{L}-\mathrm{H}$ transition takes place at $\mathrm{t}=1.40 \mathrm{~s}$, exhibiting the density rise predominantly in the edge region, and $\mathrm{H}-\mathrm{L}$ back transition at $\mathrm{t}=1.86 \mathrm{~s}$. The averaged beta suddenly increases by the transition, but saturates at about $\left\langle\beta_{\mathrm{t}}\right\rangle \sim 2.7 \%$. At the transition the $m=2 / n=3$ and $m=2 / n=2$ modes are suddenly enhanced and the former mode persists during H-phase, as shown in Fig. 2 (b). The relevant rational surface of $m=2 / n=3$ mode will be in the ergodic layer just outside LCFS. Only small ELMs are recognized during the H-phase. It is concluded that the observed beta saturation is caused by the destabilization of these low n MHD modes in the plasma edge region, of which region is in the magnetic hill. In this high beta plasma the plasma boundary seems to expand slightly more than the boundary in the vacuum configuration. This may be due to the shrinkage of the edgodic layer by a healing effect [6].

(a)

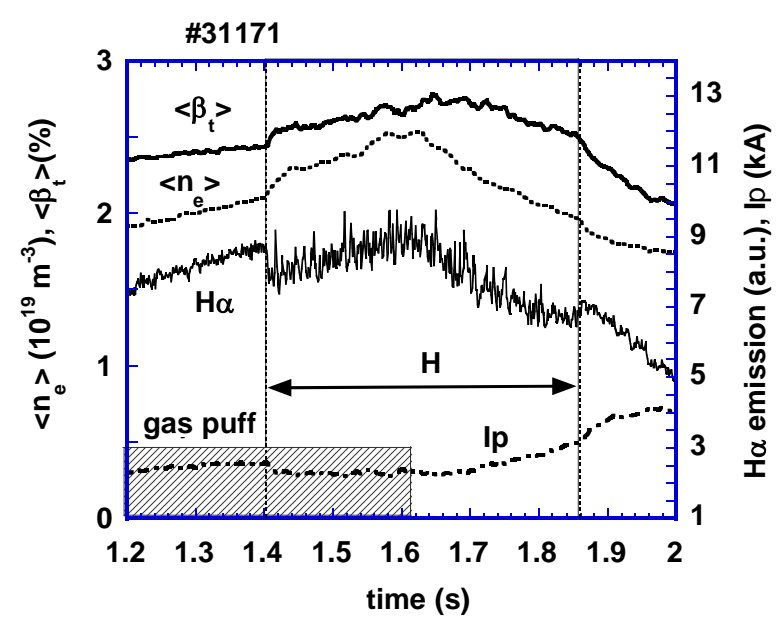

(b)

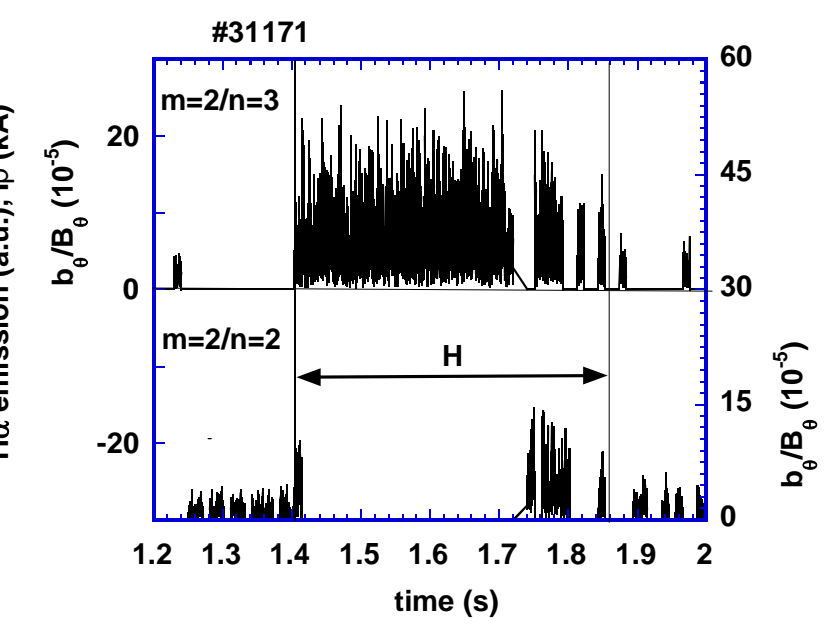

Fig.2(a) Time evolution of the averaged plasma beta obtained with diamagnetic measuremen $\left\langle\beta_{t}\right\rangle$, line averaged electron density of the center chord $\left\langle n_{e}\right\rangle$, net plasma current Ip and Ho-emission in a high beta plasma with $L-H$ transition at $B_{t}=0.5 T$ and $R_{a x}=3.6 \mathrm{~m}$. The absorbed NBI power is $P_{a b s} \sim 6$ MW. (b) Time evolution of dominant magnetic fluctuations, that is, $m=2 / n=3$ and $m=2 / n=2$ modes. 
EX/S3-2

If the edge pressure gradient is appreciably elevated having high pressure pedestal, above-mentioned edge MHD modes are also destabilized even in fairly low plasma beta of $\left\langle\beta_{\mathrm{t}}\right\rangle \leq 1 \%$ at high $\mathrm{Bt} \geq 2.7 \mathrm{~T}$. These modes are typically $m=2 / n=3$ and $m=3 / n=4$, and usually have multiple satellites. The modes are dubbed Edge Harmonic Modes (EHMs) [7]. When the plasma pressure gradient in the edge region exceeds a certain threshold, the EHMs seem to be bursting, as shown in Fig.3(a), where $\mathrm{P}_{\mathrm{abs}} \sim 5 \mathrm{MW}$ and $\mathrm{Bt}=2.75 \mathrm{~T}$. The fundamental mode of this EHM is $\mathrm{m}=3 / \mathrm{n}=4$ and the rational surface is at LCFS or in the ergodic layer. In this shot net plasma current in the co-direction reaches up to $30 \mathrm{kA}$, but does not play an essential role in converting to bursting modes. The stored energy is reduced by up to $10 \%$ by each burst. This EHM burst dominantly reduces electron density near the edge rather than electron temperature there(Figs.3(b) and (c)). Although the $m=3 / n=4$ mode would be in the very edge region, the electron density up to $\rho \sim 0.6$ is reduced by the burst. The H $\alpha$-emission exhibits very similar features to ELMs in a tokamak H-mode. Note that L-H transition does not take place in this shot. During the bursting phase of EHMs, the electron temperature profile has an island structure around $\mathrm{l}=1$ rational surface (Fig.3(b)). The bursting EHMs with $m=3 / n=4$ mode structure have obvious effect on global plasma confinement. The bursting character in EHMs is suppressed by the application of resonant helical field perturbations in order to diminish the $m=1 / n=1$ stationary magnetic island [7].

In LHD, careful tailoring of pressure and rotational transform profiles and radial electric field effects will be required to improve edge MHD stability and further increase the plasma beta $[5,8,9]$.

(a)

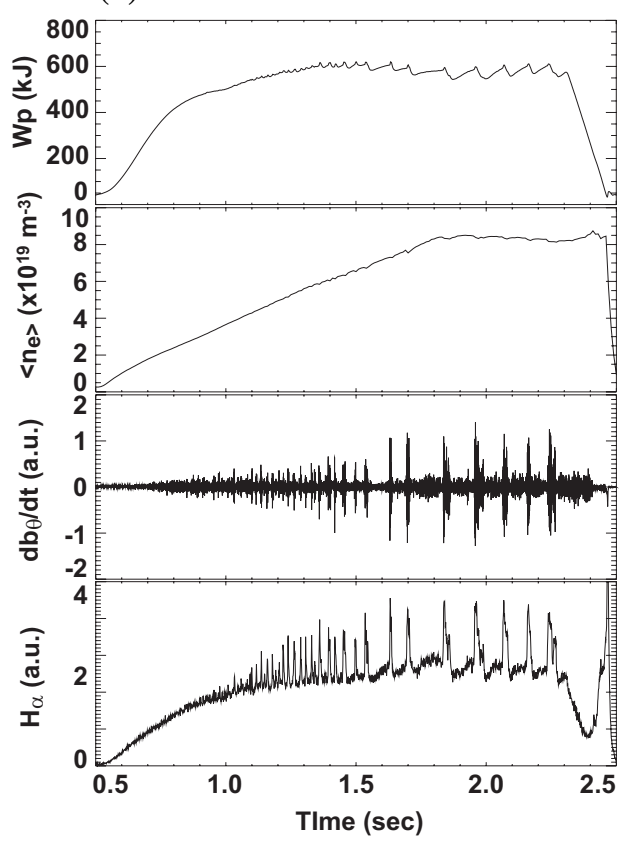

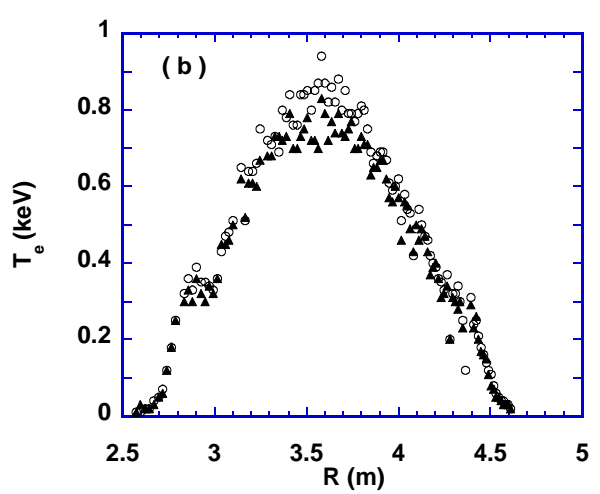

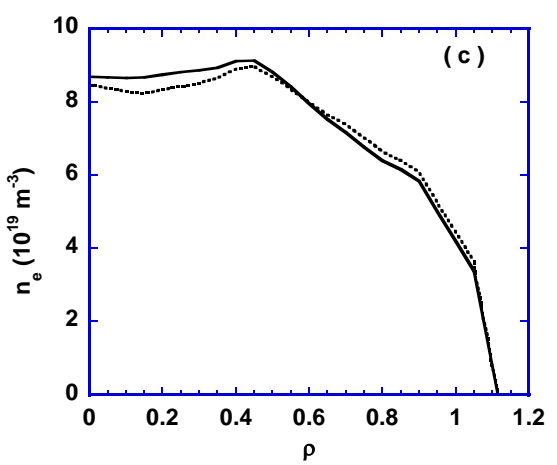

Fig.3(a) Time evolution of the stored energy, line averaged electron density, magnetic probe signal and Ho-emission in an NBI heated high density plasma with EHM bursts, where $B_{t}=2.75 \mathrm{~T}, R_{a x}=3.6 \mathrm{~m}$ and $P_{a b s}=5 \mathrm{MW}$. $($ (b) Electron temperature profiles just before and after EHM burst as a function of major radius, where open circles and solid triangles stand for Te-profiles just before and after the EHM burst, respectively. $\quad$ (c) Electron density profiles just before (dotted curve) and after (solid curve) the EHM burst. 


\section{MHD instabilities destabilized by energetic ions and their effects on plasma confinement}

The other important MHD instabilities are energetic ion driven Alfven eigenmodes such as toroidicity induced Alfven eigenmode(TAE). These MHD modes play a crucial role in realizing a fusion reactor. In major tokamaks, characteristics of AEs and their impacts on energetic ion confinement are intensively being studied, because it is thought that AEs might be destabilized by alpha particles generated by deuterium-tritium fusion reaction and would degrade the confinement and damage the first wall. This study is also important for helical systems and is continued in LHD for several years [10-12]. Excitation of AEs depends on two characteristic parameters: the averaged beta of energetic ions $\left\langle\beta_{\mathrm{b} / /}\right\rangle$ and the ratio of energetic ion velocity parallel to the magnetic field $V_{b / /}$ to the Alfven velocity $\mathrm{V}_{\mathrm{A}}$. In LHD, these two parameters are scanned over very wide range that includes those predicted for ITER[13], without suffering current disruption. Since LHD is three dimensional magnetic configuration with $l=2 / \mathrm{N}=10$ ( $l$ : field polarity, $\mathrm{N}$ : field period number), various Alfven spectral gaps are generated through toroidal mode coupling as well as poloidal mode coupling. The related gap frequency is expressed as, $f^{\mu, v}=\left|N v q^{*}-\mu\right| V_{A} /\left(4 \pi R q^{*}\right)$ where $q^{*}=(2 m+\mu) /(2 n+v N)$ is the safety factor at the relevant spectral gap, and $\nu$ and $\mu$ are the integers. The TAE corresponds to the case of $\mu=1, v=0$. The gap with $\mu=2, \nu=1$ is generated by the helical field component of $\cos (2 \theta-N \varphi)$. Helicity induced Alfven eigenmode(HAE) can be excited in the gap. As seen from the above expression, the HAE gap frequency is higher than that of TAE by a factor of $\left|N v q^{*}-\mu\right|$. It is very high for LHD of $\mathrm{N}=10$. It should be noted that the HAE frequency becomes comparable to TAE gap frequency in optimized stellarators with low N[14]. Therefore, the study of characteristics of HAEs is important for development of new helical configurations. In LHD experiments, HAEs have been detected, in addition to the observations of energetic ion driven TAEs, global Alfven eigenmodes (GAEs) and energetic particle modes(EPMs) or resonat TAEs (R-TAEs). We summarize excitation conditions of TAEs and R-TAEs in Fig.4. TAEs are excited via sideband excitation $\left(\mathrm{V}_{\mathrm{b} / /} / \mathrm{V}_{\mathrm{A}}>1 / 3\right)$ as well as funadamental excitation $\left(\mathrm{V}_{\mathrm{b} / /} / \mathrm{V}_{\mathrm{A}} \sim 1\right)$. The threshold for $\left\langle\beta_{\mathrm{b} / /}\right\rangle$ is $\sim 0.02 \%$ for TAE and $\sim 0.1 \%$ for $\mathrm{R}=\mathrm{TAE}$, where the beam beta value is estimated on the assumption of classical slowing down.
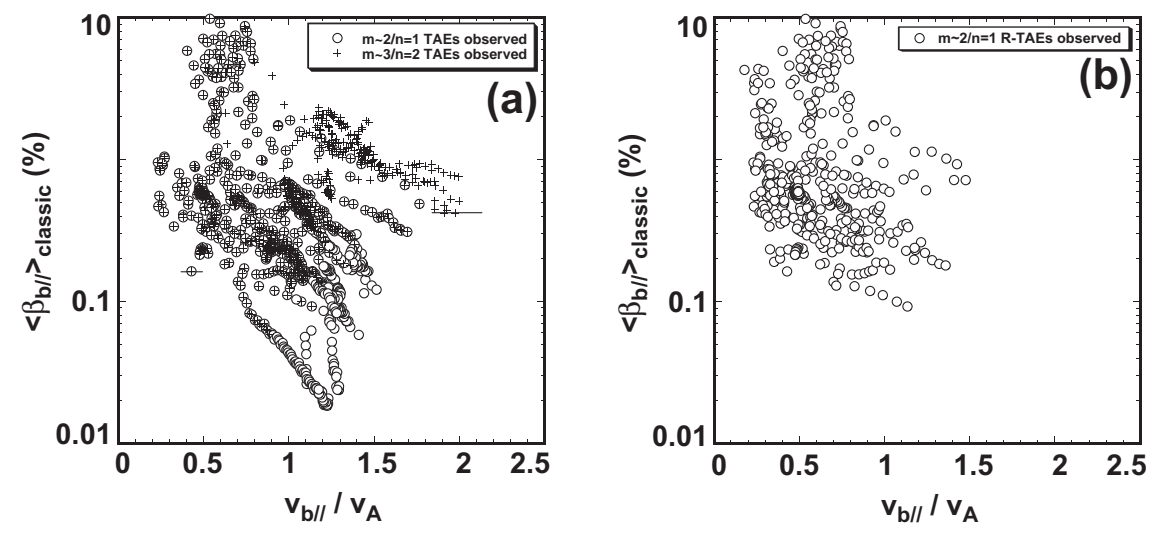

Fig.4 Parameter space for the excitation of TAEs (a) and R-TAEs (or EPMs) (b). In Fig.(a),open circles stand for $m \sim 2 / n=1$ TAE and pluses for $m \sim 3 / n=2$ TAE. In Fig.(b), $m \sim 2 / n=1$ R-TAEs are shown. 
Recently, eigenmode transition from TAE to GAE has been observed in a particular shot, where counter (ctr.)-NBI was turned off and net plasma current was increased in the co-direction[12], as shown in Fig.5. This transition is induced by the temporal evolution of the rotational transform near the plasma center, that is, the $n=1$ TAE gap generated with $m=2$ and $m=3$ mode coupling is diminished by the increase of $t(0)$ more than 0.4. Then TAE is converted to two $n=1$ GAEs with high and low frequency. The low frequency GAE will suffer from large continuum damping near the edge. As seen from Fig.5(a), the low frequency GAE quickly decays. Similar phenomena are also observed in negative shear tokamaks [15].

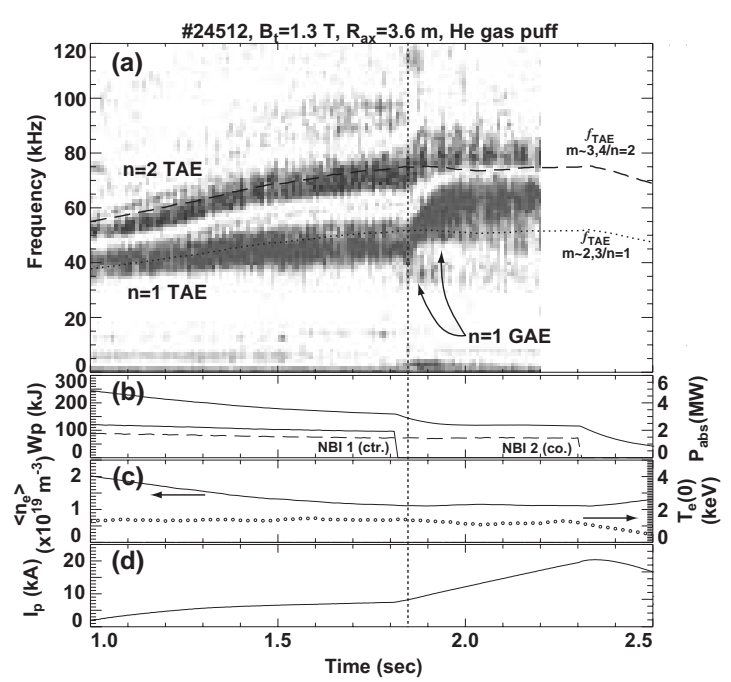

Fig.5(a) Time evolution of coherent magnetic fluctuations with $n=1$ and $n=2$ in a particular shot that eigenmode transition from TAE to GAE takes place at $t=1.83 \mathrm{~s}$. The solid and broken curves indicate the calculated TAE frequencies for $m \sim 2+3 / n=1$ and $m \sim 3+4 / n=2$ mode. The stored energy, co and counter NBI absorbed power $(b)$, the line averaged electron density, central electron temperature (c), and net plasma current (c) are also shown as a function of time.

As mentioned above, the gap frequency of HAE is higher than TAE gap frequency by about a factor of $\mathrm{N} / \mathrm{l}$, where $\mathrm{N}$ is the field period of the helical field and $\mathrm{N}=10$ for LHD. The coherent magnetic fluctuations which may be related to HAE has been detected in high beta plasmas at low toroidal field $\mathrm{B}_{\mathrm{t}} \leq 0.7 \mathrm{~T}$ (Fig.6(a))[16]. The frequency is by a factor of eight higher than the TAE gap frequency. Since the toroidal mode coupling is essential to create the HAE gap, we calculate shear Alfven spectra including 919 Fourier modes as shown in Fig.6(b)). The observed frequency resides just inside the HAE gap in the edge region. The HAE is expected to have a peak near the plasma edge. In this discharge, $n=2$ TAE related to $m=2$ and $m=3$ mode coupling is strongly excited, of which gap is around $\rho \sim 0.85$. This suggests that the energetic ion pressure profile would be broad, having appreciable gradient near the edge region. The HAE fluctuations are interrupted by this $n=2$ TAE burst. The TAE burst would enhance radial transport energetic ions near the edge, and reduce the free energy to excite HAE there. So far, this mode has no obvious effect on energetic ion confinement, because the amplitude is fairly low and frequency is high. As seen in Fig.6(a), several spectral peaks are seen between TAE and the above-mentioned high frequency mode, that is, HAE. They are thought to be induced by higher order non-uniformity of the magnetic field strength. The observation of HAE will contribute to opening new AE-physics in a toroidal plasma.

Moreover, TAEs are strongly destabilized at the low Bt plasma and become bursting. Each TAE burst has obvious impact on the signal of the stored (bulk) energy Wp measured by a diamagnetic 
EX/S3-2
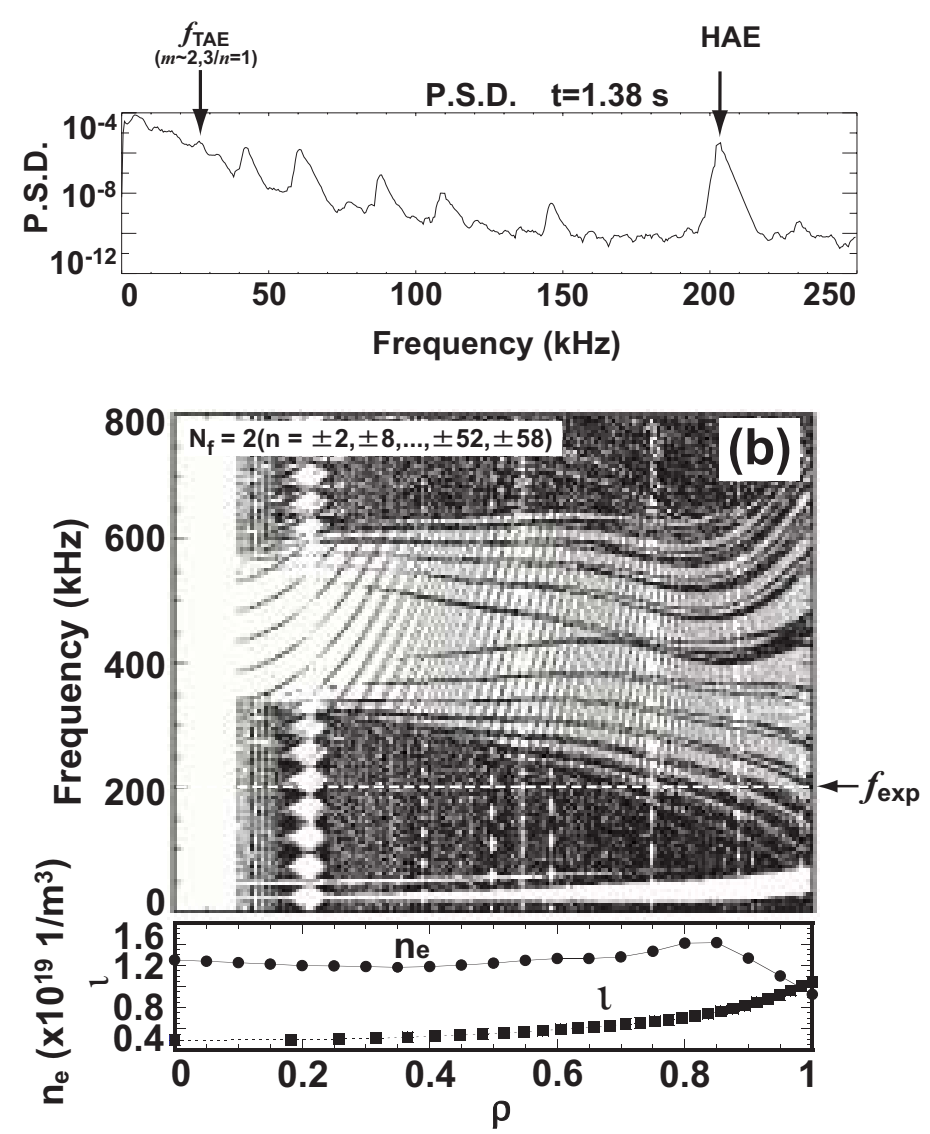

Fig.6(a) Power spectrum of magnetic probe signal. The peak at $f 200 \mathrm{kHz}$ is thought to be HAE, where TAE gap frequency is about $25 \mathrm{kHz}$. (b) $n=2$ shear Alfven spectra calculated taking account the toroidal mode coupling.

loop. By each burst, the stored energy Wp is transiently decreased by each burst as shown in Fig.7(a), where $\mathrm{Wp}$ is increased by ice pellet injection at $\mathrm{t}=0.8 \mathrm{~s}$. The characteristic decay time of $\mathrm{Wp}$ expresses the effective loss time of energetic beam ions $\tau_{\mathrm{L}}$, and is estimated to be about $3 \mathrm{~ms}$. The transient loss fraction of energetic ions is estimated to be[1-exp $\left.\left(-\delta \mathrm{t} / \tau_{\mathrm{L}}\right)\right] \sim 40-50 \%$, where $\delta \mathrm{t}$ is an effective duration of the TAE burst. In this shot, an interesting event has been recognized. The stored energy quickly recovers just after the burst, having appreciable positive value in $\mathrm{dWp} / \mathrm{dt}$ (Fig.7(b)). This can be explained by the improvement of global confinement by a factor of 1.6. The soft X-ray emissions predominantly rise in the central region, associating with each TAE burst. The line integrated electron density also increases slightly but clearly in the central chord. These results suggest a possibility that transient loss of energetic ions trigger the transient formation of internal transport barrier. A similar event induced by fishbone instability is reported from ASDEX-U [17]. This event may be related to the sheared flow generation induced through rapid radial transport of energetic ions. In this shot, the repetition frequency of bursts will not be high enough to overcome the damping rate of bulk plasma flow.

\section{Summary}

Net current free plasmas in LHD exhibit a lot of interesting features about MHD instabilities and the impacts on plasma confinement. In the high beta plasma, spontaneous L-H transition has been 
EX/S3-2
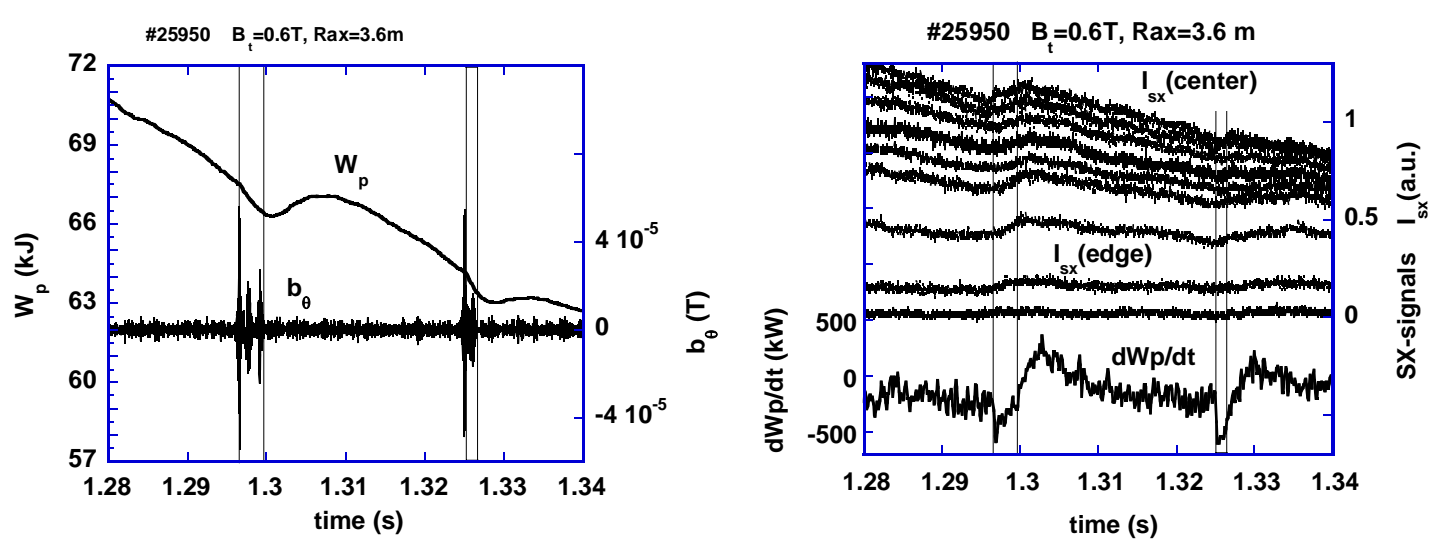

Fig.7(a) Time evolution of the stored energy in a shot with TAE bursts.

(b) Time behaviors of $d W p / d t$

and soft $X$-ray emissions in various radial locations from $\rho=0.05$ to 1.1.

observed and provides a possibility to study MHD stability in the edge region. So far, the plasma beta and global confinement are limited by the enhancement of edge MHD instabilities such as $m=2 / n=3$, although the averaged beta value exceeds $2.5 \%$. Edge MHD stability is crucial for further increasing the beta and achieve improvement of plasma confinement in LHD. It is also important to increase the pressure gradient in the core region with the help of transport barrier formation in the plasma core region where the magnetic well is created in high beta regime. In LHD, HAEs have been observed for the first time, and this observation will contribute to design studies of optimized stellarators as well as to Alfven eigenmode physics itself. In LHD, bursting TAEs transiently enhance energetic ion loss in the high beta plasma, but also trigger the transient formation of internal transport barrier.

\section{Acknowledgements}

This work was supported in part by a Grant-in-Aid for Scientific Research from Japan Society for the Promotion of Science.

\section{References}

[1] FUJIWARA, M. et al., Nucl. Fusion 41 (2001) 1355.

[2] SAKAKIBARA, S. et al., Nucl. Fusion 41 (2001) 1177.

[3] OHDACHI, S. et al., Proc. $13^{\text {th }}$ Stellarator workshop, 2002, Canberra.

[4] SAKAKIBARA, S. et al., Plasma Phys. Control. Fusion 44 (2002) A217.

[5]ICHIGUCHI, K. et al., Nucl. Fusion 41(2001) 181.

[6] NARIHARA, K., et al., Phys. Rev. Lett. 87 (2001) 135002-1.

[7] TOI, K. et al. ., $28^{\text {th }}$ EPS on Contr. Fusion and Plasma Phys. Funchal, 2001, No.P4-050.

[8] WATANABE, K. et al., Nucl. Fusion 32 (1992) 1647.

[9] SUGAMA, H. and WAKATANI, M., Phys. Fluids B 3 (1991) 1110.

[10] TOI, K. et al., Nucl. Fusion 40 (2000) 1349.

[11] TOI, K. et al., to be published in J. Plasma Fusion Res. SERIES, Vol.4(2002).

[12] YAMAMOTO, S. et al., submitted to Nucl. Fusion.

[13] ITER Physics Expert Groups, Nucl. Fusion 39 (1999) 2137.

[14] OKAMAURA, S. et al., Nucl Fusion 41 (2001) 1865.

[15] TAKECHI, M. eta al., submitted to Phys. Rev. Lett.

[16] YAMAMOTO, S. et al., to be submitted to Phys. Rev. Lett.

[17] GUENTER, S. et al., $28^{\text {th }}$ EPS on Contr. Fusion and Plasma Phys. Funchal, 2001, No.P1-006. 